EDUCATION

Research, Inovoction and Solutions on-line ${ }^{\oplus}$
PSYCHOLOGY

I+Dti
Electronic Journal of Research

in Educational Psychology

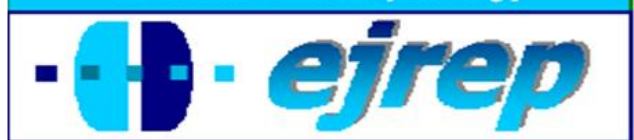

\title{
La autoevaluación sumativa en la enseñanza superior: implicaciones de su inclusión en la nota final
}

\section{Ricardo A. Tejeiro ${ }^{1}$, Jorge L. Gómez-Vallecillo ${ }^{1}$, Antonio F. Romero ${ }^{1}$, Manuel Pelegrina ${ }^{2}$, Agustín Wallace ${ }^{2}$, Enrique Emberley ${ }^{3}$,}

\author{
${ }^{1}$ Departamento de Psicología, Universidad de Cádiz, Cádiz \\ ${ }^{2}$ Departamento de Psicobiología y Metodología de las Ciencias del \\ Comportamiento, Universidad de Málaga, Málaga \\ ${ }^{3}$ Centro de Profesorado del Campo de Gibraltar, La Línea de la Concepción, \\ Cádiz
}

\section{España}

Correspondencia: Ricardo A. Tejeiro. Departamento de Psicología, Facultad de Ciencias de la Educación, Universidad de Cádiz, Campus Río San Pedro, 11519 Puerto Real, Cádiz, España. E-mail: ricardo.tejeiro@uca.es

(C) Education \& Psychology I+D+i and Editorial EOS (Spain) 


\section{Resumen}

Introducción. Se presenta un estudio que tiene por objetivo comprobar la validez de la autoevaluación sumativa criterial en la enseñanza superior y, de forma particular, si en dicha validez influye el que la autoevaluación sea considerada para elaborar la nota final.

Método. Participaron en la investigación 122 alumnos de primer curso del grado de Educación Infantil de la Universidad de Cádiz (España) pertenecientes a dos grupos diferentes, cada uno de los cuales cursaba, en el mismo periodo semestral, una asignatura distinta. La diferencia relevante entre ambos grupos era que en una de las asignaturas se incluía la autoevaluación entre los procedimientos de evaluación y se le asignaba un peso del 5\% sobre la nota final; en la otra asignatura, toda la nota era asignada por el profesor. Al concluir el período lectivo, los participantes completaron un cuestionario de autoevaluación de competencias, resultados de aprendizaje y contenidos, y se asignaron una calificación. Sus respuestas fueron comparadas con la nota asignada por el profesor, y se entrevistó a los 10 alumnos cuyas autocalificaciones mostraron mayor divergencia.

Resultados. En ambos grupos, el alumnado tendía a sobreestimar sus resultados respecto a las calificaciones asignadas por el profesor, incrementándose la diferencia de forma significativa en los alumnos con peor calificación y en el grupo cuya autocalificación influía en la nota final. En este grupo, de hecho, la calificación por el profesor y la autocalificación no guardaron ninguna relación.

Discusión. Cuando la autoevaluación no influye en la nota, el alumnado se evalúa de una manera muy similar a como lo hace el profesor, pero cuando la autoevaluación influye en la nota, la discrepancia se incrementa, notablemente, tanto por exceso como por defecto. Los principales motivos que se encuentran para ello son el deseo de obtener la máxima calificación posible y la presión añadida de tener que calificarse a uno mismo. Se analizan las implicaciones de cara a la aplicación de la autoevaluación en la enseñanza superior.

Palabras Clave: Educación; Aprendizaje; Autoevaluación; Universidad; Psicología de la Educación. 


\title{
Summative self-assessment in higher education: implica- tions of its counting towards the final mark
}

\begin{abstract}
Introduction. Our study aims at assessing the validity of sumative criterial self-assessment in higher education, and particularly if that validity varies when the teacher counts selfassessment toward final grades.
\end{abstract}

Method. One hundred and twenty two first year students from two groups in Teacher Education in Universidad de Cadiz (Spain) participated in the study, each group following a different course in the same six-month period. The significant difference between the two courses was that self-assessment was included, in one of them, among the assessment methods, and counted for 5 per cent of the final grade. The professor was the only marker in the other course. Once the courses finished, the participants completed a self-assessment questionnaire that included competences, learning results and contents, and were asked to give themselves a mark. Self-assessment data were compared with the marks given by the professor, and the 10 students with higher discrepancies were interviewed.

Results. In both groups, the students' self-assessments were higher than the grades provided by the professor, with significantly higher differences in the students with poorer results and in the group in which self-assessment influenced the final grades. In this group, in fact, no relationship was found between the professor's and the students' assessments.

Discussion. When self-assessment does not count for the final grades, students' and professor's assessments tend to be highly similar; when self-assessment counts for the final grades, over and underestimations increase dramatically. The main reasons that we found for this are the desire to obtain the highest possible grades and the stress associated with self-assessment. The implications for the implementation of self-assessment in higher education are discussed. Keywords: Education; Learning; Self-assessment; University; Educational Psychology. 


\section{Introducción}

La autoevaluación es uno de los aspectos que genera más interés en los estudios sobre la evaluación en la enseñanza superior. Los motivos para ello son variados e incluyen la búsqueda de una mayor democratización de las relaciones entre el profesorado y el alumnado, así como el fomento de la autonomía y la autorregulación como bases del aprendizaje y de la competencia profesional (Boud, 1989; Dochy, Segers y Sluijsmans, 1999; Sluijsmans, Moerkerke y Dochy, 1998).

Diversos autores defienden que la autoevaluación facilita al alumno el seguimiento, dirección y regulación de sus conductas de adquisición de información, ampliación de habilidades y automejora (Paris y Cunningham, 1996; Paris y Paris, 2001), constituyendo con ello un elemento fundamental para el aprendizaje efectivo tanto durante el periodo universitario como con posterioridad (Black y William 1998; Taras 2001). Con todo, más allá de este aparente consenso, existen numerosas definiciones diferentes y una amplia variedad de formas en que la autoevaluación es concebida y puesta en práctica.

Comenzando por el aspecto terminológico, en el ámbito anglosajón se suele distinguir entre self-assessment, self-evaluation y self-grading. El término self-assessment hace referencia al proceso de evaluación formativa que permite al alumnado reflexionar sobre la calidad de su trabajo y de su aprendizaje, juzgar el grado en que refleja los criterios establecidos para el mismo e identificar sus fortalezas y debilidades como base para su mejora (Andrade y Boulay, 2003; Goodrich, 1996; Gregory et al., 2000; Hanrahan e Isaacs, 2001; Paris y Paris, 2001; Tan, 2008). Habitualmente el self-assessment no supone la asignación de calificaciones por parte del alumno, lo que sí sucede en la self-evaluation, definida por Andrade y Du (2007) como el proceso que implica al alumnado en la realización de juicios sumativos acerca de su trabajo, que concluyen con la asignación de una nota o calificación (self-grading). En el idioma español lo habitual es encontrar de forma casi exclusiva el término autoevaluación, lo que induce a no pocas confusiones cuando no se acota su significado de forma suficiente. En este trabajo utilizaremos también el término autocalificación para hacer alusión al hecho de asignarse una nota.

Más allá de la terminología, existen notables diferencias en la forma en que se concibe la autoevaluación. Kelvin Tan entrevistó a 16 profesores de diversas disciplinas para averiguar este aspecto y halló 5 concepciones diferentes, según lo que el alumnado deba juzgar en 
cada caso (Tan, 2008): (a) su propia conducta en las actividades de autoevaluación; (b) su conocimiento de las prácticas de autoevaluación; (c) los criterios del programa de estudios; (d) su aprovechamiento dentro del programa de estudios (los juicios del alumno solo son relevantes para mejorar su aprovechamiento del programa); y (e) su capacidad de autoevaluación más allá del propio programa de estudio.

En este trabajo se presenta un caso de aplicación de la que podríamos llamar concepción "f", en la línea de Tan (2008) pero no citada por este autor: implicar al alumnado en juzgar su aprovechamiento dentro del programa de estudios de manera que su juicio repercuta de forma directa sobre su nota final. De las dos posibilidades que así se abren (que el juicio del alumno sea la única fuente de calificación o solo una parte de la nota final), veremos únicamente el segundo caso, que es el que refleja el programa docente de una de las asignaturas que cursan nuestros participantes. El caso que se comenta supone la aplicación de un procedimiento de autoevaluación criterial sumativa. El término criterial hace referencia a la existencia de un objetivo educativo especificado previamente, incluido en nuestro caso en el programa docente de la asignatura (Popham, 1983). El carácter sumativo corresponde a su aplicación al final del programa de estudio con finalidad de justificación y contabilidad (Scriven, 1967).

De forma general, la literatura asocia a este tipo de autoevaluación tanto ventajas como limitaciones. Entre las primeras, se incluye la complementación de la evaluación por el profesor, la promoción de un autoconcepto más ajustado, la facilitación en el alumno de una actitud favorable a la apertura a los juicios externos y al análisis crítico de la propia ejecución, y la reducción de la distancia social percibida entre profesor/juez y alumno/procesado. Entre las limitaciones se han citado la falta de objetividad, la tendencia a considerarse como un buen alumno o profesional, la dificultad para cuantificar la evaluación, la posibilidad de que la evaluación se convierta en una forma de autojustificación y la existencia de diferencias individuales en la capacidad para autoevaluarse (Barber, 1997).

Nuestro estudio se basa, en parte, en la comparación entre la calificación asignada por el profesor y la autocalificación, lo que constituye de hecho el procedimiento más habitual utilizado en los estudios sobre la autoevaluación (Boud y Falchikov, 1989; Brew, 1999; Falchikov, 2005; Falchikov y Boud, 1989; Gruppen et al., 1997; Hafner y Hafner, 2003). Por lo general, se ha tendido a considerar que las calificaciones asignadas por el alumno son más o 
menos válidas según su grado de semejanza con las que asigna el profesor (Tan, 2004). De la misma manera, los criterios para la bondad de la evaluación suelen ser definidos de forma exclusiva por el profesor o la institución académica (Falchikov y Boud, 1989; García y Floyd, 1999; Hanrahan e Isaacs, 2001; Longhurst y Norton, 1997), a pesar de que la investigación revela que los alumnos tienden a incluir criterios propios como el tiempo o el esfuerzo dedicado (Sullivan y Hall, 1997; Taras, 2001). Algunos autores defienden por tanto la necesidad de que tanto el profesor como el alumno participen en la determinación de los criterios (Dochy y McDowell, 1997; Stallings y Tascione, 1996).

En el presente trabajo, introducimos dos diferencias con respecto a la mayoría de la investigación previa. En primer lugar, y teniendo en cuenta que desde el inicio de la década de los 90 los perfiles profesionales y académicos tienden a definirse en términos de competencias (Lucas, 2007), se planteó una autoevaluación consistente en la autocalificación y en juicios del alumno acerca de su grado de adquisición de competencias, resultados de aprendizaje y contenidos de la asignatura. En segundo lugar, comparamos la autoevaluación que realiza el alumnado de dos grupos distintos, según la nota autoasignada repercuta o no sobre la calificación final en la asignatura. Lew et al. (2010) sugerían que, tal vez, la precisión en la autoevaluación se asocie a la forma en que el alumnado percibe el proceso, aunque en su estudio se centraban en la percepción del grado en que la autoevaluación influye sobre el aprendizaje. En nuestro caso, parece razonable suponer que los alumnos cuyos juicios influyen en su nota final, de forma directa, tenderán a estar más motivados de cara a la autoevaluación y por lo tanto la realizarán con más precisión. Somos conscientes de que existe al menos una hipótesis alternativa: que estos alumnos estarán más tentados a puntuarse de más y, por tanto, sus juicios guardarán menor relación con los del profesor.

Nuestro estudio se realiza en la Facultad de Ciencias de la Educación de la Universidad de Cádiz (España), en la que, durante el curso 2011/2012, 25 de las 61 asignaturas correspondientes a los grados incluían la autoevaluación, lo que representa el $41 \%$ del total (50\% en Educación Infantil, 42.9\% en Educación Primaria, 40\% en Actividad Física y del Deporte y $20 \%$ en Psicología). La forma en que el programa docente de cada asignatura hace referencia a la autoevaluación varía considerablemente. De forma resumida: sólo en 4 de las 25 asignaturas se especifican tareas y técnicas para llevar a cabo la autoevaluación, así como el valor cuantitativo de los resultados de cara a la calificación final del alumno. Creemos que esto refleja la falta de criterios comunes y la existencia de una cierta confusión en cuanto a la 
autoevaluación en la enseñanza superior. Diversos contactos informales con el profesorado de estas asignaturas así nos lo confirman.

\section{Objetivos e hipótesis}

Por otra parte, los estudios sobre la validez de la autoevaluación en la universidad en nuestro país son todavía escasos, como lo son, a nivel general, los que analizan el efecto que puede tener sobre la misma el que la nota autoasignada cuente o no de cara a la calificación final. Además, aunque disponemos de un buen número de investigaciones en las que se pide al alumnado que valore su aprovechamiento de una manera cuantitativa (mediante la asignación de notas) o cualitativa (argumentando sus puntos fuertes y débiles), apenas existen estudios en los que se proceda a la autoevaluación de competencias, tal como estas quedan definidas en los programas docentes.

Nuestro objetivo, por lo tanto, es cuádruple: (1) evaluar la correlación entre la autoevaluación del alumnado y las calificaciones asignadas por el profesor; (2) identificar posibles explicaciones para las diferencias entre las calificaciones asignadas por el profesor y por el propio estudiante; (3) analizar la autoevaluación directa de competencias, resultados de aprendizaje y contenidos; y (4) avanzar en el conocimiento de formas de mejorar las reflexiones y el conocimiento del alumnado acerca de su propio desempeño.

En cuanto a las hipótesis de partida, una revisión de la literatura nos lleva a plantear las siguientes: (1) las notas que se aplican los estudiantes serán similares a las asignadas por el profesor; (2) la autoasignación de una nota de 0 a 10 (procedimiento habitual de calificación durante la mayor parte de su vida académica) guardará más semejanza con la evaluación del profesor que la autoevaluación de la adquisición de competencias, resultados de aprendizaje y contenidos, al ser estos aspectos menos familiares y concretos; (3) hallaremos una mayor tendencia a sobreestimar que a subestimar los propios méritos; (4) los alumnos con mejores calificaciones evaluarán su trabajo de forma más similar al profesor que los alumnos con peores calificaciones; y (5) el que la autoevaluación influya de forma directa en la nota final se asociará a una mayor concordancia entre la nota autoasignada y la asignada por el profesor. Los trabajos que nos llevan a estas hipótesis serán comentados en la discusión de los resultados. 


\section{Método}

\section{Participantes}

Participaron en el estudio dos grupos de 60 y 62 alumnos del grado de Educación Infantil de la Facultad de Ciencias de la Educación de la Universidad de Cádiz (España). La práctica totalidad de los participantes en ambos grupos eran mujeres (95\% y 95,2\%, respectivamente) y sus edades estaban comprendidas entre 18 y 32 años $(M=20.98$; DT=3.41 у $\mathrm{M}=21.82$; $\mathrm{DT}=3.70$, respectivamente). Aunque ambos grupos pertenecen al mismo grado y tienen las mismas asignaturas, en uno de ellos (en adelante TYF) se analizó la autoevaluación en la asignatura Tutoría y Familia, y en el segundo (en adelante PSE) se hizo en la asignatura Psicología de la Educación. Ambas asignaturas se cursan durante el primer semestre del primer curso de carrera, con 14 semanas lectivas que incluyen una sesión de 2,5 horas de duración, a la que asiste todo el grupo-clase, y dos sesiones de 1 hora de duración, a cada una de las cuales asiste la mitad del grupo-clase. El profesor de las asignaturas fue el mismo (el primer autor), y también fue similar la metodología didáctica, basada en lecciones magistrales con participación activa del alumnado, prácticas individuales consistentes en búsquedas bibliográficas, y prácticas de grupo consistentes en la preparación y exposición de contenidos propios de la asignatura.

La diferencia fundamental entre ambos grupos consiste en el procedimiento de evaluación de los resultados. En el grupo PSE, la nota es asignada de forma íntegra por el profesor, ponderando el resultado del examen teórico $(60 \%)$, las prácticas $(30 \%)$ y la participación del alumnado en las clases (10\%). En el grupo TYF, la calificación incluye igualmente dichos procedimientos (con $60 \%, 30 \%$ y $5 \%$ de peso sobre la nota final, respectivamente), pero también un 5\% de autoevaluación basada, según el programa docente de la asignatura, en un "informe adicional que proporciona el alumno o el grupo respecto a su aprendizaje".

\section{Instrumentos}

Se elaboró un cuestionario de autoevaluación ad hoc en el que se pedía al alumnado que indicara, mediante una escala subjetiva de 5 puntos (desde "muy bajo" hasta "muy alto"), el grado en que creía que había adquirido cada una de las competencias, resultados de aprendizaje y contenidos relacionados en el programa de la asignatura. Cada uno de los ítems se redactó en los mismos términos en que aparece reflejado en dicho programa. En el grupo 
TYF, el cuestionario incluía 1 competencia, 4 resultados y 10 contenidos; en el grupo PSE fueron 1 competencia, 6 resultados y 9 contenidos. Asimismo, se pedía al alumnado que marcase, en una escala de 0 a 10 puntos, la calificación que, a su juicio, debería obtener en la asignatura. En el Anexo 1 se recoge el cuestionario correspondiente al grupo PSE.

\section{Procedimiento}

Los cuestionarios fueron aplicados a la conclusión del periodo lectivo, en la misma sesión del examen teórico y antes de éste. Al comienzo de la sesión, se explicó de forma breve el objetivo de los cuestionarios de autoevaluación. En el grupo PSE se señaló que el propósito era conocer mejor la percepción del alumnado sobre su propio aprendizaje a fin de optimizar futuras ediciones de la asignatura. En el grupo TYF se ofreció la misma explicación añadiendo que, de acuerdo con el programa docente, la calificación que cada uno se asignase en el ítem final supondría directamente el $5 \%$ de la nota correspondiente a la autoevaluación. Es decir, la autocalificación con un 10 supondría añadir 0.5 puntos a la calificación asignada por el profesor (que podía oscilar entre 0 y 9.5 puntos); la autocalificación con un 0 no supondría añadido alguno; y las demás notas autoasignadas supondrían un peso proporcional entre 0 y 0.5 puntos.

A fin de asegurar la independencia entre las calificaciones asignadas por el profesor y las autocalificaciones, la corrección de exámenes y la evaluación de las prácticas y de la participación en clase se llevaron a cabo antes de acceder a las respuestas a los cuestionarios de autoevaluación.

Tras el proceso de evaluación (incluida la comunicación al alumnado de sus calificaciones y la revisión de exámenes, en su caso), se mantuvieron entrevistas con los 10 alumnos con mayor discrepancia entre su autocalificación y la calificación asignada por el profesor. Las sesiones fueron grabadas en audio. El entrevistador fue el mismo profesor de la asignatura, lo que implica un cierto riesgo de deseabilidad social. Con todo, el hecho de que el entrevistador y el entrevistado hayan establecido previamente algún tipo de relación personal es considerado como un aspecto valioso en la investigación cualitativa (Rubin y Rubin, 1995). Asimismo, estimamos que los posibles efectos negativos de la deseabilidad social se minimizan por el hecho de que las entrevistas se realizasen cuando ya había concluido el semestre y 
los alumnos sabían que el profesor no volvería a darles clase en ese curso y, muy probablemente dada la estructura del grado, tampoco en el futuro.

\section{Análisis de datos}

El análisis estadístico de los datos se llevó cabo mediante el programa SPSS, versión 15.0. Para realizar los análisis estadísticos, a las calificaciones totales obtenidas por el grupo TYF se les restó la parte correspondiente a la autocalificación. Como, de esta manera, la calificación máxima posible era de 9.5 puntos, todas las notas fueron multiplicadas por 1.053 para que la calificación máxima posible fuera de 10 puntos.

Se utilizó el coeficiente de correlación $r$ de Pearson para conocer si existen diferencias entre la calificación que asigna el profesor a un alumno y la autocalificación. Dado que parte de la calificación asignada por el profesor es de carácter subjetivo (valoración de las prácticas de clase), se realizaron dos análisis sucesivos utilizando la calificación total y las puntuaciones en el examen escrito.

Las medias en calificaciones y autocalificaciones en cada grupo fueron comparadas mediante el estadístico $T$ de Student. La posibilidad de que los alumnos con mejores notas y aquellos con peores notas difiriesen en el grado de discrepancia se analizó mediante el índice de correlación entre la calificación y la discrepancia. Finalmente, para comprobar si el alumno tiende a ser más preciso en su respuesta de autoevaluación cuando ésta supone la asignación de una nota de 0 a 10 que cuando se le pide que evalúe el grado de adquisición de competencias, resultados de aprendizaje o contenidos, se sumaron las puntuaciones en los ítems relativos a resultados de aprendizaje y se agruparon en una única puntuación ponderada; lo mismo se hizo con los ítems alusivos a contenidos (en el caso de las competencias no fue necesario ya que se evaluaban con un único ítem). A continuación se calculó, en ambos grupos, la correlación entre las puntuaciones en las 3 variables resultantes, la autoevaluación y la calificación. 


\section{Resultados}

La relación entre calificación por el profesor y autocalificación fue positiva en el grupo PSE pero no en el grupo TYF, tanto cuando se consideran las calificaciones totales como cuando se analizan las puntuaciones en el examen escrito (tabla 1)

Tabla 1. Comparación de autocalificación y calificación por el profesor

\begin{tabular}{llcc}
\hline Grupo & Calificación & $\boldsymbol{r}$ & $\boldsymbol{P}$ \\
\hline \multirow{2}{*}{ TYF } & Total & .099 & .454 \\
& Examen & .270 & .838 \\
\multirow{2}{*}{ PSE } & Total & .331 & .009 \\
& Examen & .351 & .005 \\
\hline
\end{tabular}

La tabla 2 muestra las medias y desviaciones típicas de las calificaciones y autocalificaciones. En ambos grupos, la calificación que se otorgaron los alumnos fue superior a la que les asignó el profesor $(\mathrm{M}=.111, \mathrm{DT}=1,133$ para el grupo PSE; $\mathrm{M}=.280, \mathrm{DT}=1.293$ para el grupo TYF), pero en ningún caso se alcanzó la significación estadística $\left(t_{(61)}=.774, \mathrm{p}=.442\right.$ para el grupo PSE; $t_{(59)}=1.676, \mathrm{p}=.099$ para el grupo TYF).

Las discrepancias entre las autocalificaciones y las calificaciones otorgadas por el profesor variaron de $+4,1$ a $-2,4$ puntos en el grupo TYF, y entre $+2,3$ y $-1,1$ en el grupo PSE. Los alumnos con mejores y con peores notas diferían en el grado de discrepancia tanto en el grupo TYF ( $r=.705 ; \mathrm{p}=.000)$ como en el grupo PSE $(r=.473 ; \mathrm{p}=.000)$. En ambos casos, la discrepancia tendía a incrementarse (en el sentido de darse más puntuación que la consignada por el profesor) a medida que se reducía la calificación obtenida.

Tabla 2. Comparación de autocalificación y calificación por el profesor

\begin{tabular}{llcc}
\hline Grupo & Evaluador & Media & Desv.Tip. \\
\hline \multirow{2}{*}{ TYF } & Alumno & 7,72 & $(.922)$ \\
& Profesor & 7,44 & $(1,00)$ \\
\multirow{2}{*}{ PSE } & Alumno & 6,89 & $(1,05)$ \\
& Profesor & 6,78 & $(.887)$ \\
\hline
\end{tabular}

En ambos grupos, la nota final autootorgada guardaba relación significativa con la medida en que el alumno afirmaba haber adquirido competencias, resultados de aprendizaje y 
contenidos (tabla 3). En cuanto a la relación entre las autoevaluaciones de estos aspectos y la calificación otorgada por el profesor, hallamos en el grupo PSE correlaciones positivas significativas en el caso de los resultados de aprendizaje y los contenidos, pero no en el de las competencias.

Tabla 3. Relación de la calificación y la autocalificación con la autoevaluación de competencias, resultados de aprendizaje y contenidos

\begin{tabular}{|c|c|c|c|c|}
\hline Factor & Grupo & Aspecto evaluado & $\boldsymbol{r}$ & $P$ \\
\hline \multirow{6}{*}{ Calificación } & \multirow{3}{*}{ TYF } & Competencia & .172 & .189 \\
\hline & & Res. aprendizaje & .007 & .960 \\
\hline & & Contenidos & .066 & .618 \\
\hline & \multirow{4}{*}{ PSE } & Competencia & .086 & .509 \\
\hline & & Res. aprendizaje & .440 & $.000 * *$ \\
\hline & & Contenidos & .282 & $.027 *$ \\
\hline \multirow{6}{*}{ Autocalificación } & & Competencia & .377 & $.003 * *$ \\
\hline & \multirow{3}{*}{ TYF } & Res. aprendizaje & .543 & $.000 * *$ \\
\hline & & Contenidos & .594 & $.000 * *$ \\
\hline & & Competencia & .427 & $.001 * *$ \\
\hline & \multirow[t]{2}{*}{ PSE } & Res. aprendizaje & .520 & $.000 * *$ \\
\hline & & Contenidos & .499 & $.000 * *$ \\
\hline
\end{tabular}

Respecto al análisis cualitativo de los datos de las entrevistas, es destacable que los 10 alumnos con mayor diferencia entre su autocalificación y la asignada por el profesor pertenecían al grupo TYF. Los motivos alegados por los alumnos que sobrestimaron sus notas fueron: (a) dificultad para apreciar adecuadamente su aprovechamiento, (b) deseo de obtener la máxima calificación posible, (c) saber en realidad más de lo que se reflejó en el examen o (c) desconocer el motivo de la discrepancia. Los alumnos que subestimaron sus calificaciones dieron como motivos: (a) dificultad para apreciar adecuadamente su aprovechamiento, (b) deseo de evitar dar imagen de aprovechado y (c) haberse puesto nerviosos.

\section{Discusión}

¿Guardan relación las calificaciones que se aplican los estudiantes con las asignadas por el profesor? Cuando la autoevaluación no repercute en la nota final, nuestros resultados revelan que la respuesta es afirmativa. En ello nos situamos en la línea de lo ya informado por otros autores (Boud, 1986; Falchikov y Boud, 1989; Grupper et al., 1997; Hafner y Hafner, 2003; Sullivan y Hall, 1997; Taras, 2001). No se trata, sin embargo, de un resultado unánime. 
Simon Cassidy (2007) comparó las calificaciones que se asignaba una muestra de estudiantes universitarios de primer año y la que les otorgaba su profesor, hallando una correlación positiva significativa aunque relativamente baja $(r=.25)$. Este autor concluía que la mayoría de los estudiantes mostraba unas buenas habilidades de autoevaluación, que sin embargo no se manifestaban en una cuarta parte de ellos. Otras investigaciones con amplias muestras hallaron correlaciones entre sus autocalificaciones y las calificaciones del profesor en el entorno de $r=.20$ (Lew et al., 2010). En la discusión de sus resultados, afirman haber llegado a la conclusión de que "por lo general, los estudiantes muestran una ejecución bastante mala a la hora de juzgar de forma adecuada su propio proceso de aprendizaje" (Lew et al., 2010; p.147).

¿Influye la forma de autoevaluarse en la relación entre auto y heteroevaluacion? Según nuestros datos, la respuesta es negativa. En ambos grupos la autocalificación se relacionó de forma significativa con la medida en que el alumno afirmaba haber adquirido competencias, resultados de aprendizaje y contenidos. Respecto a la relación entre las autoevaluaciones de estos aspectos y la calificación otorgada por el profesor, hallamos en el grupo PSE correlaciones positivas significativas en los resultados de aprendizaje y los contenidos, pero no en las competencias. Es posible que ello se deba a los términos más generales en que estas están redactadas, o al hecho de que se evalúen con un único ítem mientras los otros aspectos se evalúan con 4-6 y 9-10 ítems.

Entre las pocas referencias a este aspecto que ofrece la literatura, destacamos el resultado de Fitzgerald, White y Gruppen (2003), quienes hallaban que los estudiantes mostraban más capacidad para autoevaluar sus conocimientos que para evaluar sus propias habilidades clínicas, aspecto un tanto más difícil de operativizar.

¿Tienden los estudiantes a sobreestimar, más que a subestimar sus méritos? Aunque se ha señalado la coincidencia significativa entre las calificaciones del alumnado y las del profesor, en ambos grupos hallamos evidencia de esta tendencia. En ello coincidimos con varios autores (Falchikov y Boud, 1989; Lew et al., 2010; Sullivan y Hall, 1997) aunque discrepamos de otros (Andrade y Du, 2007; Cassidy, 2007; Taras, 2001). Cassidy (2007), por ejemplo, cifraba la tendencia a subestimar la nota en un $56 \%$ y la tendencia a sobreestimar en un $40 \%$.

¿Son más precisos en la autoevaluación los alumnos con mejores calificaciones? Así queda de manifiesto en nuestros resultados y en los de otros estudios (Falchikov y Boud, 
1989; Lew et al., 2010; Orsmond, Merry y Reiling, 1997; Sullivan y Hall, 1997). Parece además razonable que así sea, puesto que entre los factores que influyen en la competencia de los estudiantes se incluye la capacidad para seguir y juzgar sus propios procesos de aprendizaje, ejecución, fortalezas y debilidades (Lew et al., 2010).

¿Influye sobre la precisión de la autoevaluación el hecho de que ésta cuente para la nota final? Sí, como revela la comparación de las discrepancias en las puntuaciones y el hecho de que la correlación con las calificaciones asignadas por el profesor disminuya en el grupo TYF hasta ser casi inapreciable $(r=.10)$. Sin embargo, en contra de lo esperado, esto no supone simplemente que los alumnos tiendan a puntuarse de más, ya que encontramos errores tanto por exceso como por defecto. Es decir, se produce una pérdida general de la fiabilidad de las evaluaciones.

Se ha propuesto que las bajas correlaciones entre las calificaciones de los alumnos y de los profesores puede deberse al hecho de que los primeros no tienen suficiente acceso a su propio proceso de aprendizaje (Lew et al., 2010). También la falta de experiencia en autoevaluación podría dar cuenta de una baja correlación, ya que se ha informado de que los alumnos de los últimos cursos de carrera proporcionan autoevaluaciones más precisas que los de los primeros cursos (Falchikov y Boud, 1989; Gibbs, 1995). Ambos factores son sin embargo comunes a los dos grupos de nuestro estudio: pueden por tanto haber reducido la correlación en ambos, pero no explican la diferencia entre ellos.

Para encontrar una respuesta, hemos de recurrir a los resultados de las entrevistas, que revelan dos motivos fundamentales para el aumento en la discrepancia cuando el alumno sabe que su autocalificación influirá en la nota:

(a) El deseo de incrementar su calificación. Este resultado guarda relación con el informado por Maguire, Evans y Dyas (2001), quienes hallaron que una muestra de estudiantes universitarios de primer año afrontaba las tareas de autoevaluación con un enfoque "estratégico" dirigido a obtener buenos resultados con un mínimo esfuerzo. En esta misma línea, Lew y Schmidt (2006) encontraban que muchos estudiantes creen que pueden utilizar sus autoevaluaciones para influir sobre la forma en que el profesor evalúa sus logros. Como resume un estudio: "si un profesor pide a sus alumnos que se evalúen y tiene en cuenta esas notas de cara 
a la nota final, entonces claro, los estudiantes más espabilados y motivados por las calificaciones se asignarán una A" (Andrade y Du, 2007).

(b) La presión añadida que supone el tener que calificarse a sí mismo. Taras (2001) señaló que la petición de autoevaluación genera reacciones distintas en el alumnado, con mayor preocupación entre los peores alumnos que entre los mejores. Muchos se quejan de que no poseen ni la experiencia ni los conocimientos suficientes, y manifiestan su preocupación por la posibilidad de que su autocalificación no coincida con la del profesor. Hanrahan e Isaacs (2001) destacaban la inseguridad de los alumnos en relación con los criterios de referencia, y Andrade y Du (2007) hallaban que las reacciones iniciales ante la autoevaluación incluían el malestar y la sensación de ser incapaz de llevarla a cabo. Con frecuencia, los alumnos acababan autoevaluándose en función de las expectativas que percibían en su profesor.

\section{Implicaciones para la aplicación de la autoevaluación}

El que las discrepancias se incrementen cuando la autoevaluación influye en la nota final no invalida el primero de los resultados informados: que cuando no es así, el alumnado es capaz de autoevaluarse al menos con la misma precisión con que es evaluado por sus profesores. Este resultado es relevante, en principio, de cara a la propuesta de incorporar a las aulas las actividades de autoevaluación formativa con el fin tanto de facilitar el seguimiento de sus progresos por parte de los estudiantes como de entrenar las habilidades de autoevaluación y autocontrol (Falchikov y Boud, 1989). Asumida como herramienta para que los estudiantes revisen de forma crítica su propio trabajo, la autoevaluación es por tanto factible (Andrade y $\mathrm{Du}, 2007)$. Los propios estudiantes consideran que este tipo de autoevaluación les reporta beneficios que incluyen una mejora en la comprensión del procedimiento de calificación, el desarrollo de un pensamiento crítico, el desarrollo de una mayor empatía con los profesores y un aumento de la motivación para mejorar (Hanrahan e Isaacs, 2001).

Según Kraayenoord y Paris (1997), uno de los principales objetivos de la auténtica evaluación -que incluye la autoevaluación- es animar a los estudiantes a implicarse más activamente en el seguimiento y revisión de sus propios progresos. En este sentido, los alumnos universitarios, al menos en primer curso, conservan un sólido esquema mental en el que el papel del profesor es eminentemente productivo (de contenidos, de propuestas, de retos intelectuales y de calificaciones) mientras que el del alumnado es básicamente receptivo. La 
práctica regular de autoevaluaciones formativas puede contribuir a sustituir esta concepción pasiva del rol del alumnado por un esquema más activo.

Por otro lado, si como argumentan numerosos autores el entrenamiento en la capacidad para juzgar los propios progresos supone una de las habilidades fundamentales para el aprendizaje efectivo y para el futuro desarrollo profesional (Boud, 1986; Dearing, 1997; Falchikov, 1997; Stefani, 1998; Tan, 2008), resulta si cabe más relevante en el caso de alumnos que, como es el caso de este trabajo, cursan estudios precisamente con el propósito de convertirse en maestros y maestras. Si la evaluación de progresos académicos va a suponer una parte fundamental de su futura profesión, ¿no se beneficiarían de forma particular de la práctica regular de la autoevaluación?

Algunos autores sugieren que la respuesta es negativa. Los resultados de Lew et al. (2010) parecen indicar que la autoevaluación no se aprende con la práctica, hallazgo similar al que informan Eva et al. (2004). Otros autores apuntan precisamente en sentido contrario: la autorregulación en general puede ser enseñada con instrucción explícita, con discusiones reflexivas de carácter metacognitivo y a través del modelado (Álvarez, 2009; Eissa, 2007), y Dochy, Segers y Sluijsmans (1999) hallaron que la precisión de la autoevaluación que realizaba una muestra de estudiantes mejoraba a lo largo del tiempo con la práctica.

De ser ciertos estos últimos planteamientos, es decir, de ser entrenable la autoevaluación, podemos plantearnos dos nuevas preguntas. En primer lugar, ¿cuándo deberían instaurarse estas prácticas? Taras (2001), citando también a Boud (1986) y Wood, Marshall y Hrymak (1988), sugiere que el mejor momento es el primer o segundo año de carrera, cuando el alumnado es más receptivo y cuando se puede obtener un mayor valor acumulativo. En segundo lugar, ¿cabe esperar que las habilidades de autoevaluación se generalicen de uno a otro contexto? Andrade y Du (2007) señalan en este sentido que la generalización, aún siendo posible en algunos casos, no es el resultado más habitual.

¿Debe limitarse entonces la autoevaluación a un carácter formativo, o pueden derivarse también beneficios de la autoevaluación sumativa? Uno de los principales argumentos a favor de la autoevaluación es la modificación del poder unilateral que ejerce el profesorado sobre el proceso de evaluación (Boud, 1995; Butcher y Stefani, 1995; McMahon, 1999; Rainsbury y Hodges, 1998; Somervell, 1993; Stefani, 1998). Pues bien, autores como Madda- 
lena Taras (2001) sostienen que tal modificación de las relaciones de poder no se puede alcanzar a menos que se permita al alumno realizar una autoevaluación sumativa: "si los estudiantes no tienen acceso al proceso de evaluación sumativa, su implicación en la base de poder de la educación superior será meramente periférica” (p.612). Sin embargo, Tan (2004, 2008) argumenta que la participación del alumnado en la autoevaluación sumativa solo supone un incremento en su poder cuando el resultado de esa autoevaluación prima sobre la evaluación que realiza el profesor. En esta línea, Race (1991) señala que si el estudiante sabe que su profesor no aceptará la nota que se asigna si no le resulta satisfactoria, difícilmente se puede afirmar que la autoevaluación sumativa sea participativa o redistribuya el poder.

¿Es aconsejable, por tanto, incorporar la autoevaluación sumativa y otorgarle un peso directo, total o parcial, de cara a la nota final del alumno? Nuestros resultados no apuntan en esta dirección. Este ha sido precisamente el paradigma planteado al grupo TYF, en el que se ha comprobado que, a diferencia de lo sucedido con el grupo PSE (cuya autocalificación no influía en su nota), el procedimiento de autoevaluación ha dado como resultado respuestas significativamente divergentes de las que asigna el profesor. A los argumentos ya aportados podemos añadir el de Andrade y Du (2007) de que el hecho de que la autoevaluación sea tenida en cuenta de cara a la nota final puede desviar la atención del alumnado de la calidad de su trabajo y de cómo mejorarlo, poniendo en un compromiso su honestidad y alejándolo de su objetivo de aprender.

Por otra parte, el que la autoevaluación sumativa no influya en la nota -y por tanto no redistribuya el poder, en palabras de Tan $(2004,2008)$ y Race (1991) - no le resta toda utilidad en el proceso de enseñanza-aprendizaje. Creemos que en el grupo PSE, cuya autocalificación no contaba para la nota, el haber hecho explícita la que consideraban como una calificación justa, y el hecho de que ésta coincidiera en gran medida con la que luego les asignó el profesor, contribuyó a una mayor aceptación de sus notas, traducida en una reducción de las reclamaciones posteriores al examen. Se trata, con todo, de una percepción subjetiva que no incluimos como objeto de estudio.

Probablemente la principal limitación de nuestras conclusiones proviene del hecho de que las autoevaluaciones estén siendo comparadas con el criterio del profesor y no necesariamente con el aprendizaje real del alumno, lo que resulta comprometido dada la variabilidad existente entre los profesores en cuanto a la fiabilidad con que realizan las evaluaciones. Van 
Daalen (1999) sugería que la fiabilidad y la validez en estos casos se pueden mejorar mediante el cálculo de la correlación entre la autoevaluación y el juicio de varios jueces. Este procedimiento (double-marking o multiple-marking en su terminología inglesa), no está exento de problemas (Ecclestone y Swann, 1999), pero presenta la ventaja de ser más preciso y más justo para los estudiantes, además de facilitar la coordinación de criterios entre el profesorado (Tan, 2004). En nuestro estudio, con todo, motivos de orden práctico impidieron la evaluación por varios profesores, lo que puede limitar la validez de los resultados.

Al igual que sugerían Sullivan y Hall (1997) en relación con sus hallazgos, creemos que una aportación de este estudio es la comprobación de que, aunque los alumnos en general son capaces de evaluar adecuadamente su aprovechamiento, existen numerosos casos en que se produce una discrepancia notable con la evaluación que realiza el profesor. Las respuestas a las entrevistas muestran además que el alumnado no siempre comprende el motivo de dicha diferencia, lo que puede afectar de forma negativa a su motivación, autoconcepto, actitud hacia el profesorado, etc. Si cualquier evaluación tiende a ser cuestionada en mayor o menor grado (Boud, 1995), en la educación superior, además, es necesario tener en cuenta que los alumnos se ven a sí mismos como adultos y necesitan sentir que son tratados como tal. La evaluación es un área en que se sienten particularmente vulnerables y, aunque sepan que no se les está juzgando a ellos sino a su trabajo, lo cierto es que se sienten implicados emocionalmente con el mismo y con la forma en que es valorado (Taras, 2001). Cualquier discrepancia entre la nota del profesor y la que el estudiante considera justa tiene que ser aclarada, y en este sentido la acción tutorial posterior a las calificaciones puede resultar fundamental.

Finalmente, queremos destacar que la gran preponderancia de las mujeres en nuestro alumnado ha impedido comprobar si existen diferencias de género en las respuestas de los estudiantes a la autoevaluación sumativa criterial. Se trata de un aspecto en el que aparecen resultados encontrados, tanto a favor de la existencia de tales diferencias (Andrade y Boulay, 2003; Goodrich, 1996), como en contra de las mismas (Andrade y Du, 2007; Dweck et al., 1978; Roberts y Nolen-Hoeksema, 1989), por lo que es conveniente que sea tenido en cuenta en futuras investigaciones. 


\section{Referencias}

Álvarez, I.M. (2009). Evaluar para contribuir a la autorregulación del aprendizaje. Electronic Journal of Research in Educational Psychology, 7(3), 1007-1030.

Andrade, H. y Boulay, B. (2003). Gender and the role of rubric-referenced self-assessment in learning to write, Journal of Educational Research, 97(1), 21-34.

Andrade, H. y Du, Y. (2007). Student responses to criteria referenced self-assessment. Assessment and Evaluation in Higher Education, 32(2), 159-181.

Barber, L.W. (1997). Autoevaluación. En J. Millman y L. Darling-Hammond, Manual para la evaluación del profesorado. Madrid: Editorial La Muralla.

Black, P. y William, D. (1998). Assessment and classroom learning. Assessment in Education: Principles, Policy and Practice, 5(1), 7-74.

Boud, D. (1989). The role of self assessment in student grading. Assessment \& Evaluation in Higher Education, 14(1), 20-30.

Boud, D. y Falchikov, N. (1989). Quantitative studies of student self-assessment in higher education: A critical analysis of findings. Higher Education, 18(5), 529-549.

Boud, D.J. (1986). Implementing Student Self-Assessment. Sydney: Higher Education Research and Development Society of Australia.

Brew, A. (1999). Towards autonomous assessment: using self-assessment and peer assessment. En S. Brown y A. Glasner (Eds), Assessment matters in higher education (pp. 159-171). Buckingham: SRHE y Open University Press.

Butcher, A.C. y Stefani, L.J. (1995). Analysis of peer, self- and staff-assessment in group Project work, Assessment in Education, 2(2), 165-186.

Cassidy, S. (2007). Assessing 'inexperienced' students' ability to self-assess: Exploring links with learning style and academic personal control. Assessment and Evaluation in Higher Education, 32(3), 313-330.

Dearing, R. (1997). Higher Education in the Learning Society. Londres: HMSO.

Dochy, F. y McDowell, L. (1997) Introduction: assessment as a tool for learning. Studies in Educational Evaluation, 23(4), 279-298.

Dochy, F., Segers, M. y Sluijsmans, D.M.A. (1999). The use of self-, peer and co-assessment in higher education: A review. Studies in Educational Evaluation, 24(3), 331-50.

Dweck, C., Davidson, W., Nelson, S. y Enna, B. (1978). Sex differences in learned helplessness: 2. Contingencies of evaluative feedback in the classroom and 3. An experimental analysis. Developmental Psychology, 14(3), 268-276. 
Ecclestone, K. y Swann, J. (1999). Litigation and learning tensions in improving university lecturers 'assessment practice, Assessment in Education: Principles, Policy and Practice, 6(3), 377-389.

Eissa, M.A. (2007). La eficacia de un programa basado en el desarrollo de estrategias de autorregulación de la escritura en estudiantes de educación secundaria con dificultades de escritura. Electronic Journal of Research in Educational Psychology, 7(1), 5-24.

Eva, K.W., Cunnington, J.P.W., Reiter, H.I., Keane, D.R. y Norman, G.R. (2004). How can I know what I don't know? Poor self assessment in a well-defined domain. Advances in Health Sciences Education, 9(3), 211-224.

Falchikov, N. (1997, septiembre). Why do lecturers involve students in assessment? Ponencia en la 2nd Northumbria Assessment Conference, Encouraging Partnership in Assessing Learning. University of Northumbria at Newcastle.

Falchikov, N. (2005). Improving assessment through student involvement. Abingdon, Oxon: RoutledgeFalmer.

Falchikov, N. (2005). Improving assessment through student involvement: Practical Solutions for aiding learning in higher and further education. Londres: Routledge.

Falchikov, N. y Boud, D. (1989). Student self-assessment in higher education: a metaanalysis. Review of Educational Research, 59(4), 395-430.

Fitzgerald, J.T., White, C.B. y Gruppen, L.D. (2003). A longitudinal study of self-assessment accuracy. Medical Education, 37(7), 645-649.

Garcia, J.A. y Floyd, C.E. (1999). Using single system design for student self-assessment: a method for enhancing practice and integrating curriculum. Journal of Social Work Education, 35(3), 451-461.

Gibbs, G. (1995). Assessing student centred courses. Oxford: Oxford Centre for Staff Development.

Goodrich, H. (1996). Student self-assessment: at the intersection of metacognition and authentic assessment. Tesis de Doctorado, Harvard University.

Gregory, K., Cameron, C. y Davies, A. (2000). Self-assessment and goal-setting. Merville: Connection Publishing.

Gruppen, L., Garcia, J., Grum, C., Fitzgerald, J., White, C., Dicken, L., Sisson, J. y Zweifler, A. (1997). Medical students' self-assessment accuracy in communication skills, Academic Medicine, 72(10), S57-59. 
Hafner, J. y Hafner, P. (2003) Quantitative analysis of the rubric as an assessment tool: an empirical study of student peer-group rating. International Journal of Science Education, 25(12), 1509-1528.

Hanrahan, S.J. y Isaacs, G. (2001). Assessing self- and peer-assessment: the students' views, Higher Educational Research and Development, 20(1), 53-70.

Kraayenoord, C.E.V. y Paris, S.G. (1997=. Australian students' self-appraisal of their work samples and academic progress. Elementary School Journal, 97(5), 523-537.

Lew, M.D.N. y Schmidt, H.G. (2006). Reflection upon learning between theory and practice: A focus-group study of tutors' and students' perceptions. Rotterdam: Erasmus University Rotterdam.

Lew, M.D.N., Alwis, W.A.M. y Schmidt, H.G. (2010). Accuracy of students' self-assessment and their beliefs about its utility. Assessment and Evaluation in Higher Education, $35(2), 135-156$.

Longhurst, N. \& Norton, L. S. (1997). Self-assessment in coursework essays. Studies in Educational Evaluation, 23(4), 319-330.

Lucas, S. (2007). Desarrollo de competencias desde la enseñanza universitaria. Armonización con la Enseñanza Secundaria y el mercado de trabajo, desde la Psicología Social de la Educación. Electronic Journal of Research in Educational Psychology, 5(1), 1696-2095.

Maguire, S., Evans, S.E. y Dyas, L. (2001). Approaches to learning: A study of firstyear geography undergraduates. Journal of Geography in Higher Education, 25(1), 95-107.

McMahon, T. (1999). Using negotiation in summative assessment to encourage critical thinking. Teaching in Higher Education, 4(4), 549-554.

Orsmond, P., Merry, S. y Reiling, K. (1997). Students and tutors perceptions of a good essay. Research in Education, 58, 81-84.

Paris, S.G. y Cunningham, A.E. (1996). Children becoming students. En D.C. Berliner y R.C. Calfee (Eds.), Handbook of educational psychology (pp. 117-147). Nueva York: Simon \& Schuster Macmillan.

Paris, S.G. y Paris, A.H. (2001). Classroom applications of research on self-regulated learning, Educational Psychologist, 36(2), 89-101.

Popham, W.J. (1983). Problemas y técnicas de evaluación educativa. Madrid: Anaya.

Race, P. (1991). Learning through assessing. Comunicación presentada en la Standing Conference on Educational Development. Newcastle, Reino Unido. 
Rainsbury, E. y Hodges, D. (1998). Academic, employer and student collaborative assessment in a work-based cooperative education course, Assessment and Evaluation in Higher Education, 23(3), 313-325.

Roberts, T. y Nolen-Hoeksema, S. (1989). Sex differences in reactions to evaluative feedback, Sex Roles, 21(11-12), 725-746.

Rubin, H. y Rubin, I. (1995). Qualitative interviewing: the art of hearing data. Thousand Oaks, CA: Sage.

Scriven, M.S. (1967). The methodology of evaluation, en Perspectives of curriculum evaluation (AERA Monograph Series on Curriculum Evaluation, $n^{\circ} 1$ ). Chicago: Rand McNally.

Sluijsmans, D.M.A., Moerkerke, G. y Dochy, F.J.R.C. (1998). Creating a learning environment by using self-, peer- and co-assessment. Learning Environments Research, 1, 293319.

Somervell, H. (1993). Issues in assessment, enterprise and higher education: the case for self-, peer and collaborative assessment. Assessment and Evaluation in Higher Education, $18(3), 221-233$.

Stallings, V. y Tascione, C. (1996) Student self-assessment and self-evaluation, Mathematics Teacher, 89(7), 548-555.

Stefani, L.J. (1998). Assessment in partnership with learners, Assessment and Evaluation in Higher Education, 23(4), 339-350.

Stefani, L.J. (1998). Assessment in partnership with learners, Assessment and Evaluation in Higher Education, 23(4), 339-350.

Sullivan, K. y Hall, C. (1997). Introducing students to self-assessment. Assessment and Evaluation in Higher Education, 22(3), 289-305.

Tan, K.H.K. (2004). Does student self-assessment empower or discipline students? Assessment and Evaluation in Higher Education, 29(6), 651-662.

Tan, K.H.K. (2008). Qualitatively different ways of experiencing student self-assessment. Higher Education Research and Development, 27(1), 15-29.

Taras, M. (2001). The Use of Tutor Feedback and Student Self-assessment in Summative Assessment Tasks: towards transparency for students and for tutors. Assessment and Evaluation in Higher Education, 26(6), 605-614.

Van Daalen, M. (1999). Test usefulness in alternative assessment. Dialog on Language Instruction, (1-2), 1-26. 
Wood, D.R., Marshall, R.R. y Hrymak, A.N. (1988). Self-assessment in the context of the McMaster Problem Solving Programme. Assessment and Evaluation in Higher Education, 13(2), 107-127.

Anexo I. Cuestionario de autoevaluación correspondiente al grupo PSE.

\section{UNIVERSIDAD DE CÁDIZ; GRADO EN EDUCACIÓN INFANTIL PSICOLOGÍA DE LA EDUCACIÓN PRUEBA DE AUTOEVALUACIÓN}

NOMBRE FECHA

1. Indica con una $X$ el grado en que crees que has adquirido la siguiente competencia:

\begin{tabular}{|l|l|l|l|l|l|}
\cline { 2 - 5 } \multicolumn{1}{c|}{} & $\begin{array}{c}\text { Muy } \\
\text { bajo }\end{array}$ & Bajo & Medio & Alto & Muy alto \\
\hline $\begin{array}{l}\text { Comprender los procesos edu- } \\
\text { cativos y de aprendizaje rela- } \\
\text { tivos en el período de 0-6 } \\
\text { años, en el contexto familiar, } \\
\text { social y escolar }\end{array}$ & & & & & \\
\hline
\end{tabular}

2. Marca con una $\mathbf{X}$ el grado en que crees que has alcanzado cada uno de los siguientes resultados de aprendizaje:

\begin{tabular}{|l|l|l|l|l|l|}
\cline { 2 - 5 } \multicolumn{1}{l|}{} & $\begin{array}{c}\text { Muy } \\
\text { bajo }\end{array}$ & Bajo & Medio & Alto & Muy alto \\
\hline $\begin{array}{l}\text { Comprender la familia como } \\
\text { contexto de aprendizaje y la } \\
\text { necesidad de colaboración con } \\
\text { la escuela }\end{array}$ & & & & & \\
\hline $\begin{array}{l}\text { Comprender la importancia de } \\
\text { los procesos de percepción en } \\
\text { la atención temprana. }\end{array}$ & & & & & \\
\hline $\begin{array}{l}\text { Conocer y establecer relacio- } \\
\text { nes entre el aprendizaje, los } \\
\text { procesos psicológicos básicos } \\
\text { y el proceso educativo en } \\
\text { Educación Infantil. }\end{array}$ & & & & & \\
\hline $\begin{array}{l}\text { Elaborar propuestas de actua- } \\
\text { ción basadas en tales conoci- }\end{array}$ & & & & & \\
\hline
\end{tabular}




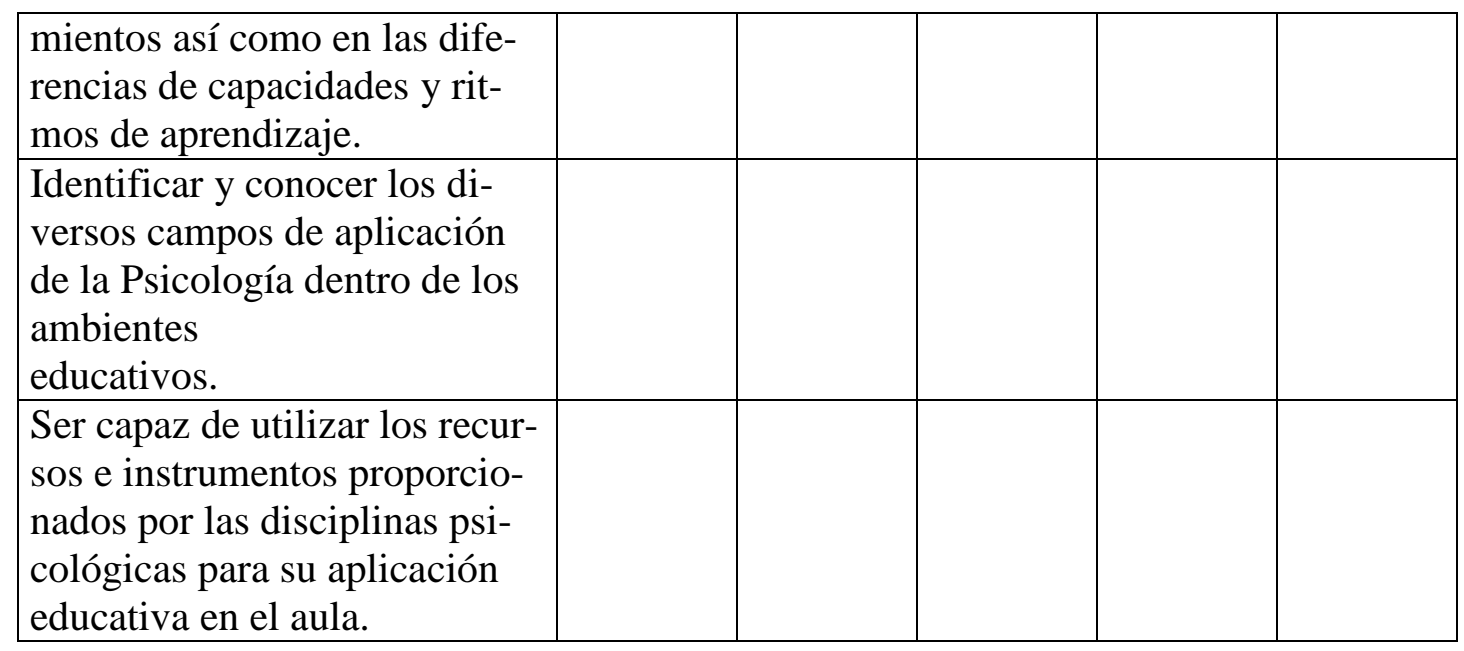

3. Señala con una $X$ el grado en que crees que has aprendido los contenidos que se relacionan a continuación:

\begin{tabular}{|c|c|c|c|c|c|}
\hline & $\begin{array}{l}\text { Muy } \\
\text { bajo }\end{array}$ & Bajo & Medio & Alto & Muy alto \\
\hline $\begin{array}{l}\text { La Psicología de la Educación } \\
\text { en el contexto de la ciencia } \\
\text { psicológica. }\end{array}$ & & & & & \\
\hline $\begin{array}{l}\text { Modelos psicológicos del pro- } \\
\text { ceso de enseñanza-aprendizaje } \\
\text { aplicados al ámbito de la Edu- } \\
\text { cación Infantil y de la } \\
\text { atención temprana }\end{array}$ & & & & & \\
\hline $\begin{array}{l}\text { Procesos psicológicos básicos } \\
\text { en Educación Infantil }\end{array}$ & & & & & \\
\hline $\begin{array}{l}\text { La construcción del conoci- } \\
\text { miento en el aula }\end{array}$ & & & & & \\
\hline $\begin{array}{l}\text { Interacción en el aula y su } \\
\text { repercusión en ámbito social, } \\
\text { afectivo y de aprendizaje en } \\
\text { educación infantil }\end{array}$ & & & & & \\
\hline $\begin{array}{l}\text { La familia como contexto de } \\
\text { aprendizaje }\end{array}$ & & & & & \\
\hline $\begin{array}{l}\text { El método científico y sus } \\
\text { aplicaciones }\end{array}$ & & & & & \\
\hline $\begin{array}{l}\text { Estrategias en el aprendizaje } \\
\text { eficaz }\end{array}$ & & & & & \\
\hline $\begin{array}{l}\text { Valores y creencias familiares } \\
\text { sobre la educación }\end{array}$ & & & & & \\
\hline
\end{tabular}

4. Rodea con un círculo la calificación que, a tu juicio, deberías obtener en esta asignatura:

\begin{tabular}{|l|l|l|l|l|l|l|l|l|l|l|}
\hline 0 & 1 & 2 & 3 & 4 & 5 & 6 & 7 & 8 & 9 & 10 \\
\hline
\end{tabular}

